\title{
The Inhibition of Fusarium oxysporum var. cubense by Musarin, an Antibiotic produced by Meredith's Actinomycete
}

\author{
By H. R. V. ARNSTEIN, A. H. COOK AND MARGARET S. LACEY \\ The Imperial College of Science and Technology, London
}

SUMMARY: Under selected conditions Meredith's actinomycete produced culture fluids toxic to many fungi. From these fluids the active principle, called musarin, has been isolated by two methods. Preparations active in concentrations of $1 / 80,000-1 / 100,000$ seemed substantially pure.

Musarin is an optically active acid of high molecular weight having by microtitrationan equivalent weight of at least 4000. Analyses of the best preparations are in good agreement with an empirical formula $\left(\mathrm{C}_{35} \mathrm{H}_{60} \mathrm{O}_{14} \mathrm{~N}_{2}\right)_{72}$. No definite conclusion can be drawn about the exact chemical nature of musarin; a protein-like structure seems to be excluded by the low nitrogen content, which recalls that of many bacterial polysaccharides; but formulation as a polysaccharide seems equally unsatisfactory because of the low oxygen content.

Tests indicate that musarin is one of the most potent antifungal antibiotics, though as an antibacterial agent it is less effective. It is active against several important plant parasites and it may be useful in combating the Panama disease of the banana plant and other fungal diseases of economic importance.

The so-called Panama disease of the banana plant (Musa sapientum), caused by Fusarium oxysporum var. cubense, is known to be inhibited by certain Jamaican soils. From such soils Meredith (1943, 1944) isolated actinomycetes which were able to inhibit or retard the development of $F$. oxysporum var. cubense. Later it was shown that Meredith's actinomycete produced soluble antibiotics against $\boldsymbol{F}$. oxysporum, particularly when grown on food yeast waste liquor (Thaysen \& Butlin, 1945) or on meat extract media (Thaysen \& Morris, 1947).

Whilst the biological aspects of the problem are being further investigated by $\operatorname{Dr} \mathrm{A}$. C. Thaysen in the Colonial Microbiological Research Institute, Trinidad, we record here a study of the production, isolation and properties of a new antibiotic obtained from the culture filtrate of the actinomycete, and termed for convenience musarin. This name, although recalling the host plant rather than the parent organism, seems justified as it avoids confusion with antibiotics derived from other actinomycetes.

\section{EXPERIMENTAL}

\section{Cultural characters of Meredith's actinomycete}

The original culture of Meredith's actinomycete grown on peptone or synthetic agar media was dry, wrinkled and uniformly white, but on malt-extract agar the cultures became deeply pigmented, pale to deep yellow, red, green and a few chalk-white colonies appearing in a single culture. When malt-agar 
plates were inoculated from these variously pigmented growths it was found that all the young colonies were white or yellow, the red and green pigments developing later in some of the yellow colonies. Green colonies often became dark red, almost black, after several days; but some colonies started turning red on the third day of growth. By selection and successive replating of the earliest red colonies a culture in which every colony turned red was finally obtained. By similar selection, homogeneous chalk-white and deep-yellow cultures were also obtained; but since the red strain was found to give the best results the other strains were not investigated in detail. The red strain was, however, unstable and had to be continuously selected. Moreover, the colour of the colonies was not a sure indication of the capacity to produce active solutions when grown on liquid media and the above relationship of colour and activity changed after repeated transfers. It was noticed, however, that active strains invariably produced a dark, diffusible pigment on both malt-agar and in liquid casein media, although the pigment had no other connexion with the active principle; musarin itself is colourless.

\section{Methods of testing for antibiotic activity}

For the routine examination of actinomyces culture liquids for antifungal activity $F$. lateritium was used as the test organism in preference to $F$. oxysporum var. cubense, since it readily produces masses of spores.

(a) Agar-plate method. Malt-extract agar (for fungi) or peptone Lemco agar (for bacteria) was seeded with the test organism and plates were poured. The antibiotic liquids were filled into holes cut in the medium with a cork-borer $1 \mathrm{~cm}$. diameter. The width of the inhibition zone was read after overnight incubation at $37^{\circ}$ for bacterial plates. Fungal plates were incubated at $\mathbf{2 4}^{\circ}$, and 2 or occasionally 3 days' incubation was necessary before results were obtained. The width of the zone varied with potency of the fluid. A second, and sometimes a third, ring or partial inhibition usually developed beyond the completely clear zone, frequently edged with a narrow ring of stimulated growth.

(b) A serial broth dilution method was also employed for antibacterial tests.

(c) Hanging-drop method for anti-spore germination tests. A suspension of Fusarium spores in nutrient broth was mixed with an equal quantity of solution or with serial dilutions of antibiotic liquids and hanging-drop slides were prepared from the mixtures. Results were read after overnight incubation at $24^{\circ}$. The antifungal titre of the solution was taken as the highest dilution completely inhibiting spore germination $(++++)$. One unit of antibiotic potency is arbitrarily defined as the amount of musarin which in $1 \mathrm{ml}$. of nutrient medium completely inhibits $F$. lateritium spore germination. The following degrees of partial inhibition were also noted: (1) emergence of germ tubes from some of the spores but no further growth $(+++) ;(2)$ germination of the spores but no normal mycelial growth, yeast-like budding $(++)$; (3) limited mycelial growth followed by formation of masses of microconidia $(+)$. 
The width of the inhibition zone obtained by method $(a)$ was closely correlated with the degree of inhibition obtained by method $(c)$ in parallel tests of the same culture filtrate. Roughly,,,,++++++++++ corresponded to titres of $c .1 / 2,1 / 4,1 / 8$ and $1 / 16$ respectively, as estimated by method $(\dot{d})$.

(d) Dilution plates for tests of antifungal activity. Solutions of musarin were added to measured quantities of melted malt-extract agar to give final dilutions of $1 / 50,000,1 / 100,000$, etc., and the mixture was poured into Petri dishes. A loopful of a spore suspension of the fungus was placed on the surface of each dilution plate. The plates were incubated at $24^{\circ}$ and examined for spore germination and mycelial growth at intervals up to 6 days. In most cases there was good mycelial growth on the control plate after $\mathbf{4 0} \mathrm{hr}$. In the antifungal tests the results given by methods $(a)$ and $(d)$ and in a limited number of tests by method $(c)$, were closely correlated.

\section{Production of musarin}

Musarin was detected by its antibiotic activity against $\boldsymbol{F}$. lateritium spores, tested as described above. It was produced when the actinomycete was grown in stationary, or shaken (submerged) cultures (see below); it is possible that under different conditions more than one antibiotic was formed but the stability and biological properties of the active culture fluids suggested that the antibiotics were at least very similar.

Stationary cultures. The actinomycete was grown in $150 \mathrm{ml}$. conical flasks containing $40 \mathrm{ml}$. of a defined medium, consisting of $\mathrm{KCl} 0.5 \mathrm{~g}$., $\mathrm{MgSO}_{4} .7 \mathrm{H}_{2} \mathrm{O}$ $0.5 \mathrm{~g}$., $\mathrm{FeSO}_{4} .7 \mathrm{H}_{2} \mathrm{O} 0.01 \mathrm{~g}$., and $\mathrm{K}_{2} \mathrm{HPO}_{4} 1.0 \mathrm{~g}$., in $1 \mathrm{l}$. of water to which was added yeast extract, malt extract, Eupepton No. 2, Bacto-Tryptone or casein, either with or without $1 \%$ glucose. With the exception of the yeast-extract medium without sugar and the malt-extract medium with sugar, cultures in all the media were active against $\boldsymbol{F}$. lateritium. The greatest activity was obtained when I \% glucose and either $0.5 \%$ casein or $0.5 \%$ Eupepton were added. Using the original mixed actinomycete culture, the fluid after 12 days at $25^{\circ}$ contained 8 units $/ \mathrm{ml}$. with strong retardation of mycelial growth. It also completely inhibited the growth of Mycobacterium phlei at 1/50, and was fairly active against Staphylococcus aureus and Bacillus subtilis, but only feebly active against Bacterium coli and Pseudomonas pyocyanea (Ps. aeruginosa).

In one experiment, a flask was coated on the inside with a thin layer of malt-extract agar, and $40 \mathrm{ml}$. of Eupepton-glucose solution were added after the agar had set. The actinomycete grew well in the solution and formed a thick ring of growth on the agar at the surface of the fluid, which became deeply pigmented. This culture contained 8 units/ml. but caused slight inhibition at $1 / 64$, and a very definite clear zone, $8 \mathrm{~mm}$. wide, on a malt-agar plate seeded with Fusarium lateritium (method $a$ ). Similar results were obtained on Lemco peptone-agar slopes with solutions of $1 \%$ glucose, lactose or sucrose broth at the bottom of the tubes, the sucrose culture being the most active.

When four flasks were inoculated respectively with the red, yellow and white 
strains and the original (mixed) culture, the medium containing $\mathrm{NaCl} 5 \mathrm{~g}$., $\mathrm{K}_{2} \mathrm{HPO}_{4} 1.0 \mathrm{~g}$., glucose $10 \mathrm{~g}$., and casein $5 \mathrm{~g}$., in $1 \mathrm{l}$., all except the yellow strain produced dark brown pigment. Antibiotics against $F$. lateritium spores appeared most rapidly (11 days) in the red culture, slowly in the original and white strains and very slowly in the yellow, but the final maximum titre was the same in all four cultures. All four cultures were equally active against Myco. phlei on the 20th day (1/25) but were inactive against Bact. coli. The white culture alone feebly inhibited Streptococcus pyogenes and was also slightly more active than the other three against Staph. aureus and B. subtilis.

In a second series of tests the red, white and yellow strains were inoculated into flasks, the interior of which had been coated with Lemco peptone-agar, containing $18 \mathrm{ml}$. of liquid casein-glucose medium. Again the red strain produced dark brown pigmentation of both the agar and the liquid but the yellow culture did not do so; the white culture slowly produced pigmentation of the agar but the liquid remained uncoloured. As before the red culture produced the antibiotic most rapidly, though on this occasion the white was slower than the yellow strain. In general, activities against a variety of organisms were similar to those in the previous experiment except that the red cultures were active against Bact. coli whereas the white and yellow strains were inactive; moreover, the red strain was as active as the white against Strep. pyogenes whereas the yellow was inactive.

All three cultures were very active against Verticillium albo-atrum and $V$. dahliae; the diameter of the inhibition zone (method $a$ ) on malt-agar plates was $18 \mathrm{~mm}$.

Production in quart-size milk bottles containing. $400 \mathrm{ml}$. of medium was unsuccessful. With a medium similar to that used for the production of streptothricin by Streptomyces lavendulae (Waksman, 1943), containing $\mathrm{K}_{2} \mathrm{HPO}_{4} 0.5$ g., $\mathrm{NaCl} 0.5$ g., $\mathrm{FeSO}_{4} .7 \mathrm{H}_{2} \mathrm{O} 0.01$ g., glucose $10 \mathrm{~g}$., and BactoTryptone $5 \mathrm{~g}$. in $1 \mathrm{l}$. of water, antibiotic was produced only when the organism could be induced to grow on the surface by providing support in the form of pieces of porous porcelain. With a starch $(2 \%)$ glycine $(1 \%)$ medium (Waksman, 1943) no antibiotic was obtained, probably for the same reason.

The active fluid from stationary cultures was unaffected by acid ( $\mathrm{pH} \mathrm{2 \cdot 4)}$ or alkali ( $\mathrm{pH} \mathrm{10.5)}$ at room temperature for $30 \mathrm{~min}$. but was completely inactivated at $100^{\circ}$.

Shaken cultures. Shaken cultures of the above Bacto-Tryptone glucose medium, using the original culture of the actinomycete, produced antibiotic after 14 days (2 units/ml.). Eupepton, glycine and corn-steep liquor were less effective in that order. The antibiotic so produced was as stable as that in stationary culture fluids. Heating at $100^{\circ}$ at $\mathrm{pH} 7$ for $30 \mathrm{~min}$. inactivated $50 \%$.

Submerged cultures. The fermentation vessel (Fig. 1) was a tank of 1 in. plate iron 15 in. high and 16 in. diameter, designed so that when charged with medium and assembled the whole could be sterilized as a unit. It was fitted with a stout screw-on iron lid and rubber washer; the lid was removable for cleaning but carried a screw-on cap $(C)$ for charging, inoculating, and sampling, and a metal stirrer $(S)$ the paddles of which closely fitted the vessel. The tank 
was provided with an inlet $(I)$ connected to a circular iron tube drilled in the form of a gas-ring burner and reaching almost to the bottom of the tank. When in operation the stirrer was mechanically driven and sterilized air was injected through the inlet $I$ by a pump so that the medium was thoroughly aerated. The tank was used about half-full with a charge of about $20 \mathrm{l}$. No special temperature control was necessary, but good results were obtained only when the room temperature was $20-25^{\circ}$. With a small excess pressure inside the apparatus and a tight-fitting stirrer gland and cotton-wool packing at $C$ during sterilization, no contamination occurred. The iron scaled during use; this was not harmful, and plating the tank with stainless steel had no marked advantage.

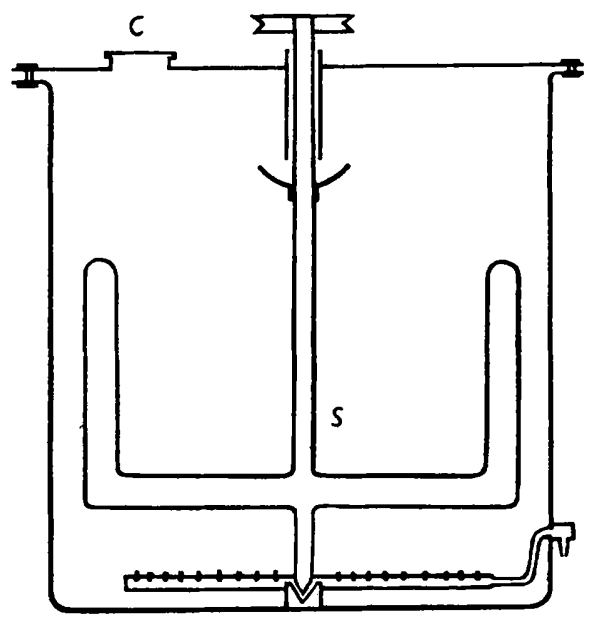

Fig. 1. Fermentation vessel.

Inoculations were made with a single-slope culture of actinomycete, previously macerated with water. Rapid fermentation, especially in presence of higher concentrations of Bacto-Tryptone, caused much inconvenient frothing through the loosely fitting sampling lid $C$. The concentration of BactoTryptone, which seemed to be the important nutrient for antibiotic production, was therefore chosen so that fermentation and antibiotic production took about 4 days. More rapid production was followed by more rapid destruction of the antibiotic by aeration and it was found impracticable in view of the methods of assay to estimate the time of peak activity in such rapid fermentations. It was also found advantageous to decrease the rate of aeration to the minimum and to limit the amount of medium to about $17 \mathrm{l}$. in order to avoid frothing.

Submerged cultures of the original mixed strain were grown in the tank using a medium containing $\mathrm{NaCl} 0.05 \%, \mathrm{~K}_{2} \mathrm{HPO}_{4} 0.05 \%, \mathrm{FeSO}_{4} .7 \mathrm{H}_{2} \mathrm{O}$ $0.0025 \%$, Eupepton $0.25 \%$, Bacto-Tryptone $0.25 \%$, and glucose $1 \%$. After 4-5 days the solution contained 32 units $/ \mathrm{ml}$., but after 6 days the potency had dropped to 8 units $/ \mathrm{ml}$. With $1 \%$ Bacto-Tryptone fermentation was very rapid and although the culture was slightly active after 2 days, the activity soon 
disappeared. When Bacto-Tryptone was replaced by Eupepton the maximum production of antibiotic was 2 units $/ \mathrm{ml}$.

The red strain of the actinomycete was found to produce as much antibiotic as the mixed culture, but the yellow strain produced little or none with either Bacto-Tryptone $(0.5 \%)$ or Bacto-Tryptone $(0.25 \%)$ and Eupepton $(0.25 \%)$ (max. 2 units $/ \mathrm{ml}$.). Growth of the white strain on the standard medium (Bacto-Tryptone 0.25\%, Eupepton 0.25\%) was slower than with the red; its cultures consistently contained 16 units $/ \mathrm{ml}$. after 4 days, dropping to 8 units $/ \mathrm{ml}$. after 5 days. The red strain, giving 32-64 units $/ \mathrm{ml}$, was therefore selected for further study. The effect of changing some of the constituents of the standard medium is shown in Table 1, which indicates that the standard medium had the most favourable composition for antibiotic production by the red strain.

Table 1. Effect of variation of medium on the antibiotic produr
by the red strain of Meredith's actinomycete
$\begin{aligned} & \text { Component varied } \\ & \text { Bacto-'Tryptone }(0 \cdot 375 \%)\end{aligned}$
$\begin{aligned} & \text { Bacto-Tryptone }(0 \cdot 25 \%) \\ & \text { Eupepton nil }\end{aligned}$
$\begin{aligned} & \text { Bacto-Tryptone }(0 \cdot 375 \%) \\ & \text { Eupepton }(0 \cdot 125 \%)\end{aligned}$
$\begin{aligned} & \text { Glucose }(1 \cdot 5 \%) \\ & \text { Standard medium }\end{aligned}$

\section{Isolation of musarin from the fermented tank solution}

Early experiments with stationary culture fluids showed that the only solvent suitable for extracting musarin was $n$-butanol at $\mathrm{pH} 7$. Extraction of a shaken culture by this means removed the antibiotic activity completely. The butanol layer was separated, concentrated in vacuo, and treated with excess of ether, when an almost white solid precipitated. The solid was filtered off; it was slightly hygroscopic and turned brown in air. This material contained 35 units/mg. This method failed with large-scale extractions because the antibiotic produced by submerged fermentation was thermolabile. The thermolability proved to be due to the impurities in the culture fluid, since purified preparations were found to be relatively thermostable. It was hoped to back-extract the active material into water by adding ether ( 5 vol.) or light petroleum ( 5 vol.) but the partition coefficients were unfavourable, only 66 and $\mathbf{5 0} \%$, respectively, of the activity being recovered in the aqueous layer. The antibiotic was adsorbed on charcoal $(2 \%)$ and partially on fuller's earth (1 \%) but could not be satisfactorily eluted. In this respect, as in many others to be described later, musarin differs from streptomycin and streptothricin, which can be eluted from charcoal with acid alcohol (Le Page \& Campbell, 1946; Peck, Walti, Grater, Flynn, Hoffhine, Allfrey \& Folkers, 1946). Experiments with precipitating reagents showed that musarin was precipitated by adding ammonium sulphate almost to saturation; $64 \%$ of the activity could be recovered from the precipitate by extraction with water and only $47 \%$ by extraction with methanol. Musarin was partially precipitated from the culture 
fluid by $\mathrm{NaCl}$, but not by $\mathrm{Ca}_{3}\left(\mathrm{PO}_{4}\right)_{2}$. The addition of acid to $\mathrm{pH} 3$ also precipitated most of the active material. Two methods of isolating musarin are described here.

(1) To 20 l. culture filtrate at $0^{\circ}$ containing 160,000 units was added $9 \mathrm{~kg}$. ammonium sulphate. The solution was allowed to stand for $2 \mathrm{hr}$. and the precipitate (A) was filtered off; on further standing more solid (B) precipitated. A cold methanol extract of precipitate $A$, after concentration in vacuo and precipitation with ether, yielded $933 \mathrm{mg}$. of a white solid (20 units/mg.; 18,500 units). A second extraction with hot methanol yielded $1178 \mathrm{mg}$. of a solid containing 47,100 units. Precipitate B (10 g.; 100,000 units), treated in a similar way, yielded $187 \mathrm{mg}$. (7000 units) and $239 \mathrm{mg}$. (2400 units) respectively. The total amount of musarin recovered was therefore 75,000 units, corresponding to a recovery of $47 \%$. In addition, $203 \mathrm{mg}$. of a solid containing 16,000 units were obtained from the air-dried mycelium by methanol extraction. An aqueous solution of any of these materials gave a precipitate with mercuric chloride or copper acetate solution but no active fraction was recovered. Solution of the less active solids in $1 \%$ neutral potassium phosphate buffer and addition of $2 \mathrm{~N}-\mathrm{H}_{2} \mathrm{SO}_{4}$ to $\mathrm{pH} 3$ gave a precipitate, which was separated by centrifuging and washed with acetone and ether. This solid, which decomposed at about $170^{\circ}$, contained 80 units $/ \mathrm{mg}$. It contained neither halogen nor sulphur; it was insoluble in water but dissolved readily in $1 \%$ neutral phosphate buffer.

(2) The following method gave better results and was used for routine isolation of musarin.

The tank culture fluid was freed from all solid matter by decanting and filtration. The filtrate (15 l., containing 480,000 units) was acidified to $\mathrm{pH}$ 3.5-4.0 with dilute $\mathrm{H}_{3} \mathrm{PO}_{4}$. The addition of $c .0 \cdot 5 \%$ neutral alumina or silica before acidification at this point often facilitated the subsequent filtration but was not essential. A precipitate appeared almost immediately and was allowed to settle. The solution was then filtered and the filtrate, containing 60,000 units, was discarded. The precipitate was eluted by shaking vigorously for $1 \mathrm{hr}$. with 1.61. $1 \%$ neutral sodium phosphate buffer solution. The eluate was filtered $(320,000$ units; recovery, $67 \%)$ and extracted twice with $500 \mathrm{ml} . n$-butanol. After the first extraction 32,000 units (10\%) and after the second extraction only 3200 units $(1 \%)$ remained in the aqueous layer. The butanol extracts were combined, washed with a little water, and evaporated to dryness in vacuo at $45^{\circ}$. The residue was dissolved in $c .100 \mathrm{ml}$. of methanol and the solution filtered and concentrated to small volume in vacuo. Addition of ether precipitated 2 g. of a slightly yellow solid, containing 100 units $/ \mathrm{mg}$. The recovery was therefore at least 200,000 units or $42 \%$. Some of the yellow pigment could be removed by reprecipitating the antibiotic from methanol with acetone, but no increase in potency was achieved by this treatment.

The mycelium of the actinomycete also yielded musarin, though the quantity isolated was somewhat variable and the potency was usually low. In one instance the air-dried mycelium from $20 \mathrm{l}$. of fermented solution was extracted with hot methanol (wt. after extraction $=7 \cdot 7$ g.). The solution was filtered and 
evaporated to dryness under reduced pressure. The residue was dissolved in $1 \%$ neutral phosphate buffer and extracted with $n$-butanol. The butanol extract was concentrated in vacuo. Addition of ether precipitated $203 \mathrm{mg}$. of an almost white solid, which contained 80 units/mg.

\section{Chemical properties of musarin}

Musarin is an acid with an equivalent weight of $c .5000$ as indicated by electrometric titration. The sodium or potassium salt is soluble in water, methanol, ethanol and butanol, but not in ether or acetone. The free acid is insoluble in water or acetone, but is soluble in $30 \%$ aqueous acetone and alcohol. The acid is obtained as a flocculent precipitate when a neutral solution of musarin is acidified with dilute $\mathrm{HCl}, \mathrm{H}_{3} \mathrm{PO}_{4}$ or $\mathrm{H}_{2} \mathrm{SO}_{4}$, but organic acids such as acetic or oxalic acid do not precipitate musarin completely. Musarin is also precipitated by $\mathrm{BaCl}_{2}, \mathrm{HgCl}_{2}$ or copper acetate, but the active material cannot be recovered from the precipitates or the supernatant liquid.

The free acid is somewhat unstable even when kept as the dry solid, but the sodium salt appears to be stable. The activity was unaltered at $\mathrm{pH} 2$ or 11 at room temperature for $30 \mathrm{~min}$. but was completely destroyed at $100^{\circ}$ under these conditions. Heating at $100^{\circ}$ at $\mathrm{pH} 7$ caused $50 \%$ inactivation after $30 \mathrm{~min}$. and $60-80 \%$ inactivation after $1 \mathrm{hr}$.

Table 2. Chromatography of musarin on alumina

$\begin{array}{cccc}\text { Fraction } & \begin{array}{c}\text { Vol. of eluate } \\ \text { (ml.) }\end{array} & \begin{array}{c}\text { Wt. of solid } \\ \text { (mg.) }\end{array} & \text { Total units } \\ 1 & 35 & 20 & 80 \\ 2 & 30 & 143 & 3,600 \\ 3 & 20 & 335 & 27,000 \\ 4 & 35 & 270 & 21,500 \\ 5 & 110 & 70 & 4,500 \\ 6 & 80 & \text { Trace } & -\end{array}$

Fraction 3: $[\alpha]_{D}^{24}=32 \cdot 3^{\circ} \pm 2 \cdot 4^{\circ}$. Recovery of active material from whole chromato$\operatorname{gram}=33 \%$.

Musarin (80-100 units/mg.) could not be purified further by fractional precipitation with $0.0506 \mathrm{~N}-\mathrm{HCl}$ or by chromatography on alumina. Chromatography was best carried out by using Merck's alumina which was adjusted to pH 5-6 with $50 \% \mathrm{H}_{2} \mathrm{SO}_{4}$ and washed thoroughly with $85 \%$ aqueous methanol. In a typical experiment $2 \mathrm{~g}$. of musarin $[\alpha]_{D}^{20^{\circ}}=35 \cdot 1^{\circ} \pm 1 \cdot 6^{\circ}$ (80 units/mg.) in $85 \%$ aqueous methanol ( $50 \mathrm{ml}$.) were purified on $20 \mathrm{~g}$. of Merck's alumina. The column was eluted with $85 \%$ aqueous methanol. Each fraction was concentrated in vacuo to 5-10 ml. and the active material was precipitated with c. $100 \mathrm{ml}$. acetone (Table 2)

The most potent preparations of musarin sodium salt decomposed at about $170^{\circ}$ without melting. The sodium salt was optically active $[\alpha]_{D}^{20^{\circ}}=35 \cdot 1^{\circ} \pm 1 \cdot 6^{\circ}$ (c. 1.21 in methanol); $[\alpha]_{D}^{200}=38 \cdot 7^{\circ} \pm 2 \cdot 7^{\circ}($ c. 0.736 in methanol) and absorbed

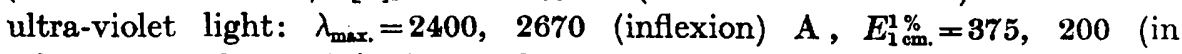
ethanol). Analyses of the free acid (Found: C, 57.75; H, 8.34; N, 3.70\%) and 
of the sodium salt of similar potency (Found: C, 57.5, 56.8; H, 8.55, 8.27; $\mathrm{N}, 4.08,4.25 \%$ ) were very similar, reflecting the high equivalent weight of musarin. Musarin did not contain sulphur, halogen or phosphorus. These data are in agreement with an empirical formula, $\mathrm{C}_{35} \mathrm{H}_{60} \mathrm{O}_{14} \mathrm{~N}_{2}$ (requires $\mathrm{C}, 57 \cdot 35$; $\mathrm{H}, 8 \cdot 2 ; \mathrm{N}, 3 \cdot 8 \%$ ).

Musarin gave negative Molisch, Millon and biuret tests for carbohydrate and protein respectively, but certain preparations gave a weak ninhydrin test after hydrolysis with $5 \mathrm{~N}-\mathrm{HCl}$ at $100^{\circ}$ for $2 \mathrm{~min}$. Since this reaction was not given by all preparations it is not conșidered significant. Musarin gave no coloration with sulphuric acid in acetic acid or with iodine, and the Salkowski and Liebermann tests for steroids and cholesterol as well as the murexide test were also negative. Musarin did, however, give a positive Axenfeld reaction for protein, i.e. a red colour was obtained when a solution of gold chloride was added to a boiling solution previously acidified with formic acid; on addition of gold chloride a blue-violet colour developed.

Treatment of musarin with an ethereal solution of diazomethane resulted in an inactive product which was insoluble in $1 \%$ neutral phosphate buffer. Similarly, treatment with methanol containing a few drops of methanolic $\mathrm{HCl}$ gave an inactive product, soluble in acetone and chloroform but insoluble in $1 \%$ neutral phosphate buffer. This solid had an optical rotation $[\alpha]_{D}^{22^{\circ}}=\mathbf{3 2} \cdot \mathbf{2} \pm \mathbf{2 \cdot 0 ^ { \circ }}$ (c. 0.995 in methanol).

Titration of musarin with acid and alkali. Micro-titration both of the free acid with sodium hydroxide and hydrochloric acid and of musarin sodium salt with hydrochloric acid was carried out in a special cell using a glass electrode (Catch, Cook \& Kitchener, 1945). Titration of the free acid (40 units/mg.) indicated an equivalent weight of $c$. 8000, but of this musarin sodium salt (80 units/mg.), a value of $c$. 4000. The titration curves were complex, presumably due to the presence of other groups.

\section{Biological properties of musarin}

In all the following tests musarin containing 80 or 100 units/mg. was used.

Antifungal activity. Table 3 records the highest dilutions of musarin in which the germination of the spores of sixteen fungal species was completely inhibited. In most cases the next higher dilution delayed germination for 24-48 hr. and there was corresponding poor and slow mycelial development. In the lower dilutions most of the spores looked dead but no tests were made to prove this. The germination of spores of Fusarium lini was permanently inhibited only at $1 / 50,000$ but in $1 / 100,000$, although the spores germinated, they produced only short, hypertrophied germ-tubes which shrivelled and died. A dilution of musarin of $1 / 200,000$ caused delay and poor development of mycelial growth in $F$. lini. It is apparent from Table 3 that musarin is a powerful antifungal agent, being especially active against the destructive plant parasites Verticillium dahliae, V. albo-atrum, Botrytis cinerea and Ceratostomella paradoxa. 
Comparison of the antifungal activity of musarin with streptomycin and streptothricin. The antifungal activity of musarin was compared with that of a sample of streptomycin containing 700 units (Schatz, Bugie \& Waksman, 1944) per mg. and of a sample of streptothricin containing 400 units (Foster \& Woodruff, 1943) per mg., against the sixteen fungal species given in Table 3

Table 3. Antifungal activity of musarin

(80-100 units/mg.)

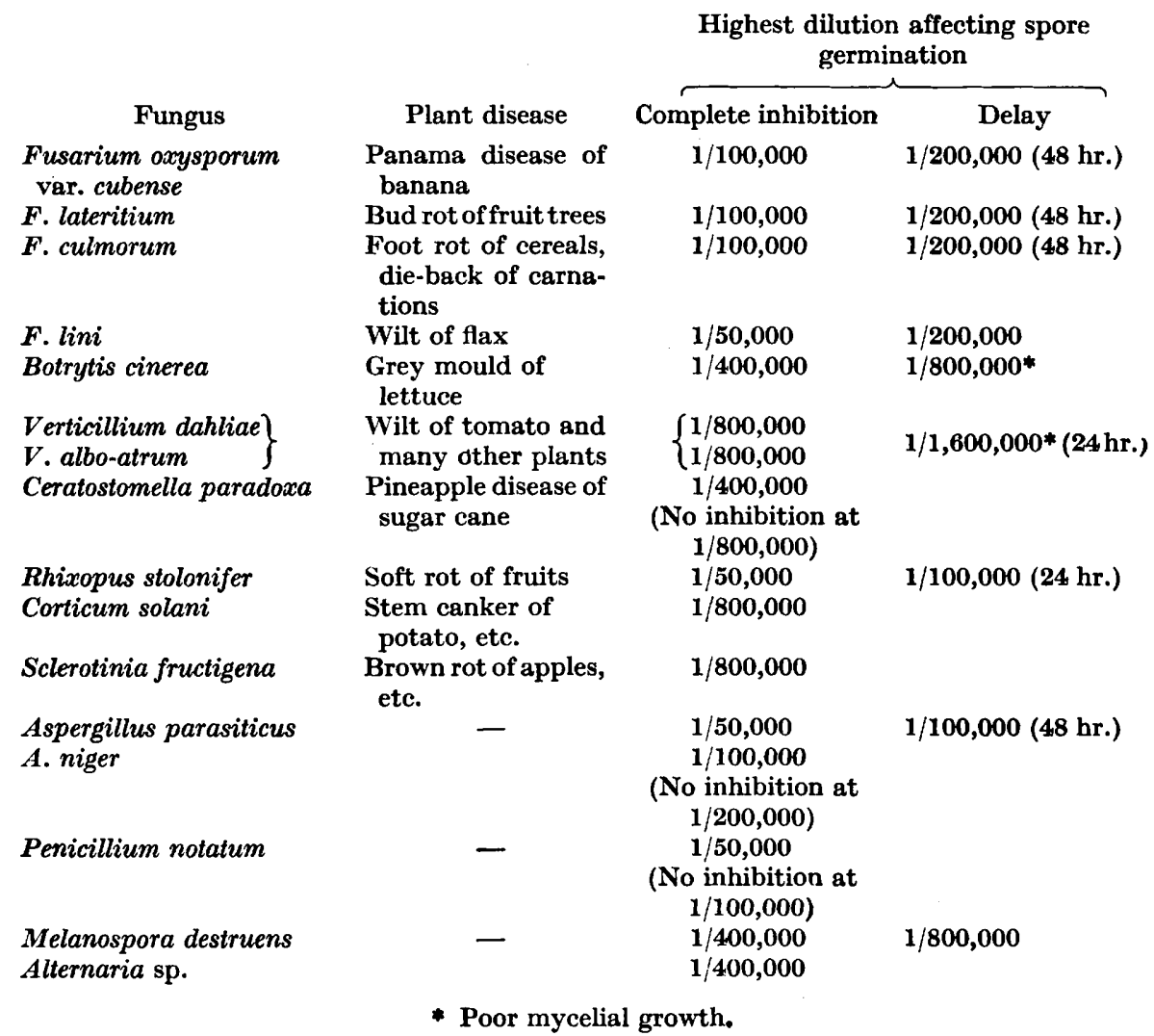

(method $a$ ). Streptomycin was quite inactive against all the fungi and a concentration of $1 / 100$ did not inhibit the germination of Fusarium lateritium spores in hanging-drop cultures.

Streptothricin was active against all the fungi except Aspergillus parasiticus. It was about as potent as musarin against the four Fusarium strains, Penicillium notatum and Aspergillus niger, but was much less active against the other nine cultures; moreover, the inhibition of all growth in and over the surface of the clear zones was permanent on the musarin test plates but on the streptothricin plates the mycelial growth gradually spread all over the originally inhibited areas. For example, musarin produced a $15 \mathrm{~mm}$. wide zone against 
Ceratostomella paradoxa and, this area was still entirely free from growth $\mathbf{2 5}$ days later; streptothricin produced a $10 \mathrm{~mm}$. wide zone which was reduced in 3 days to $3 \mathrm{~mm}$. by the growth of mycelium over the surface.

Antibacterial activity of musarin. Musarin, in a concentration of $1 / 500$, produced a $7 \mathrm{~mm}$. wide inhibition zone in plates of Staph. aureus. From these tests and from similar tests against $B$. subtilis and Strep. pyogenes it appeared that musarin was only feebly active against Gram-positive bacteria; but when broth dilutions were made it was found that musarin was very active against Staph. aureus, completely inhibiting growth at $1 / 500,000$. The highest effective dilutions of musarin against five bacterial species is shown in Table 4 .

Table 4. Antibacterial activity of musarin (80-100 units/mg.)

$\begin{array}{lc}\text { Bacterium } & \begin{array}{c}\text { Highest effective } \\ \text { dilution }\end{array} \\ \text { Staph. aureus } & 1 / 500,000 \\ \text { B. subtilis } & 1 / 100,000 \\ \text { Strep. pyogenes } & 1 / \mathbf{2 5 , 0 0 0} \\ \text { Myco. phlei I } & 1 / 100,000 \\ \text { Bact. coli } & \text { Less than } 1 / 5000\end{array}$

It was evident that the agar-plate method gave no criterion of the antibacterial activity of musarin, although it gave a fairly accurate measure of antifungal activity. Two explanations of the divergence of results seemed possible: musarin might diffuse into an acid but not into an alkaline medium, for whereas the fungal tests were made on acid malt-agar, the bacterial tests

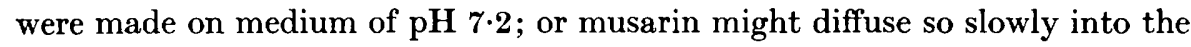
medium that the rapidly growing Staph. aureus colonies were established before the antibiotic had reached beyond the $7 \mathrm{~mm}$. zone. Owing to the slower rate of growth of fungi, the fungal plates would not be so affected by this factor. Experiments showed that the second explanation was correct. Thus, a $15 \mathrm{~mm}$. wide zone developed round a 1/1000 solution of musarin on a test plate of Staph. aureus held at room temperature, whereas a control plate, incubated at $37^{\circ}$ produced the usual $7 \mathrm{~mm}$. zone only.

Comparison of the antibacterial activity of musarin, streptomycin and streptothricin. Broth dilution tests showed that streptomycin and streptothricin were much more active than musarin against Myco. phlei. The limiting titre of musarin was $1 / 100,000$, but streptomycin (700 units/mg.) and streptothricin (400 units/mg.) completely inhibited the growth of $M y c o$. phlei at 1/250,000, the highest dilution tested. Musarin was more active against Staph. aureus than either streptomycin or streptothricin. All three antibiotics were about equally active against Strep. pyogenes but streptomycin was less active against $B$. subtilis than either musarin or streptothricin. The greatest difference, however, in antibacterial activity was against $\boldsymbol{B}$ act. coli, for whereas both streptomycin and streptothricin were effective against this organism in about $1 / 75,000$ dilution, there was heavy growth of Bact. coli even in the 1/5000 dilutions of musarin (Table 5). 
Table 5. Antibiotic activity of musarin (80-100 units/mg.), streptomycin (700 Waksman units/mg.) and streptothricin (400 Foster \& Woodruff units/mg.)

\begin{tabular}{|c|c|c|c|}
\hline \multirow[b]{2}{*}{ Test organism } & \multirow[b]{2}{*}{ Musarin } & \multicolumn{2}{|c|}{ Highest effective dilution of } \\
\hline & & Streptomycin & Streptothricin \\
\hline General antifungal activity & Very strong, permanent & None & Strong, transient \\
\hline Aspergillus parasiticus & $1 / 50,000$ & None & None \\
\hline Verticillium sp. & $1 / 800,000$ & None & $1 / 250,000$ \\
\hline Khizoctonia sp. & $1 / 800,000$ & None & $1 / 50,000$ \\
\hline Cerátostomella sp. & $1 / 400,000$ & None & $1 / 50,000$ \\
\hline Staph. aureus & $1 / 500,000$ & $1 / 100,000$ & $1 / 100,000$ \\
\hline Myco. phlei I & $1 / 100,000$ & $<1 / 250,000$ & $<1 / 250,000$ \\
\hline Bact. coli & $>1 / 5000$ & $1 / 75,000$ & $1 / 75,000$ \\
\hline
\end{tabular}

We are indebted to Sir Ian Heilbron, D.S.O., F.R.S., for his interest and encouragement and to the Colonial Products Research Council, under whose auspices this work was carried out, for assistance. We also thank Dr A. C. Thaysen for the original actinomycete cultures used in this work.

\section{REFERENCES}

CATCh, J. R., Cook, A. H. \& KITChener, J. A. (1945). Apparatus for micro-titrations with a glass electrode. J. chem. Soc. p. 319.

Foster, J. W. \& Woodruff, H. B. (1943). Quantitative estimation of streptothricin. J. Bact. 45, 408.

Le Page, G. A. \& Campbell, E. (1946). Preparation of streptomycin. J. biol. Chem. $162,163$.

Meredith, C. H. (1943). The antagonism of actinomyces to Fusarium oxysporum cubense. Phytopath. 33, 403.

Meredith, C. H. (1944). The antagonism of soil organisms to Fusarium oxysporum cubense. Phytopath. 34, 426.

Peck, R. L., Walti, A., Grater, R. P., Flynn, E., Hoffhine, C. E., Allfrey, V. \& Folkers, K. (1846). Streptomyces antibiotics. VI. Isolation of streptothricin. J. Amer. chem. Soc. 68, 772.

Schatz, A., Bugie, E. \& Waksman, S. A. (1944). Streptomycin, a substance exhibiting antibiotic activity against Gram-positive and Gram-negative bacteria. Proc. Soc. exp. Biol., N.Y., 55, 66.

Thaysen, A. C. \& Butlin, K. R. (1945). Inhibition of the development of Fusarium oxysporum cubense by a growth substance produced by Meredith's actinomycete. Nature, Lond., 156, 781.

Thaysen, A. C. \& Morris, M. (1947). Medium suitable for the cultivation of Meredith's actinomycete. Nature, Lond., 159, 100.

Waksman, S. A. (1943). Production and activity of streptomycin. J. Bact. 46, 299. 